\title{
ON UNIFORM LIMITEDNESS OF SETS OF FUNCTIONAL OPERATIONS *
}

BY T. H. HILDEBRANDT

1. Introduction. F. Riesz $\dagger$ has pointed out that the method of proving the Hellinger-Toeplitz theorem is closely related to certain proofs of boundedness of sequences of integrals. Essentially these state that a sequence of limited linear operations on a given class, bounded for every member of the class, is uniformly limited. The Hellinger-Toeplitz theorem is a special case of the theorem that a double sequence of limited bilinear forms, if bounded for every member of the class, is uniformly limited. The question naturally arises whether a theorem of the second type can be derived from a theorem of the first type by an iterative process. This paper derives such a theorem of the first type, by extracting the essential feature of the linearity condition, and utilizing some of the fundamental ideas of E. H. Moore and H. L. Smith.

2. The Foundations. We shall assume a general class $\mathfrak{P}$ of elements $p$; also a class $\mathfrak{M}$ of functions $\mu$ on $\mathfrak{B}$ to $\mathfrak{A}$ the class of complex numbers. The class $\mathfrak{M}$ is assumed to be linear (L), i.e., for every $a_{1}$ and $a_{2}$ of $\mathfrak{A}$ and $\mu_{1}$ and $\mu_{2}$ of $\mathfrak{M}$, $a_{1} \mu_{1}+a_{2} \mu_{2}$ also belongs to $\mathfrak{M}$. We assume further that it is possible to define in $\mathfrak{M}$ a distance function or norm $N$, which is on $\mathfrak{M}$ to the real positive part of $\mathfrak{A}$ and has the property $(\Delta)$ : for every $a_{1}$ and $a_{2}$, and $\mu_{1}$ and $\mu_{2}$

$$
N\left(a_{1} \mu_{1}+a_{2} \mu_{2}\right) \leqq\left|a_{1}\right| N \mu_{1}+\left|a_{2}\right| N \mu_{2} . \S
$$

In terms of this norm it is possible to define the notion

* Part of a paper presented to Society, March 26, 1921.

$\dagger$ † Équations Linéaires à une Infinité d'Inconnues, footnote, p. 83.

$\ddagger$ General theory of limits, AMerican Journal, vol. 44 (1922), pp. $102 \mathrm{ff}$.

$\S$ This condition $(\Delta)$ is equivalent to the two conditions

$$
N\left(\mu_{1}+\mu_{2}\right) \leqq N \mu_{1}+N \mu_{2}, \quad N a \mu_{1}=|a| N \mu_{1}
$$

for every $\mu_{1}, \mu_{2}$ and $a$. For from $N a \mu \leqq|a| N \mu$ it follows for $a \not 00$ that

$$
N a \mu \leqq|a| N \mu=|a| N\left(\frac{a}{a} \mu\right) \leqq \frac{|a|}{|a|} N a \mu=N a \mu \text {. }
$$


distance as $N\left(\mu_{1}-\mu_{2}\right)$ as well as limit, viz.,

$$
\lim _{n} N\left(\mu_{n}-\mu\right)=0 \text {. }
$$

In so far as we do not postulate that $N \mu=0$ if and only if $\mu=0$ for every $p$, the limit of a sequence $\mu_{n}$ thus defined is not necessarily unique. If two functions $\mu_{0}$ and $\mu_{00}$ are limits of the same sequence then obviously $N\left(\mu_{0}-\mu_{00}\right)=0$.

We shall call a Cauchy sequence a sequence $\left\{\mu_{n}\right\}$ such that for every $e>0$ there exists an $n_{e}$ such that if $n_{1} \geqq n_{e}$ and $n_{2} \geqq n_{e}$, then

$$
N\left(\mu_{n_{1}}-\mu_{n_{2}}\right) \leqq e .
$$

We shall assume that the class $\mathfrak{M}$ is complete $(\mathrm{Cp})$ with respect to $N$, viz., that every Cauchy sequence from $\mathfrak{M}$ has a limit in $\mathfrak{M}$.*

Finally we use some of the ideas suggested by E. H. Moore and H. L. Smith. We assume another general class: $\mathfrak{Q}$ of elements $q$. On $\mathfrak{Q}$ we assume a binary relation $R: q_{1} R q_{2}$, with the properties

$(T)$ if $q_{1} R q_{2}$ and $q_{2} R q_{3}$ then $q_{1} R q_{3}$;

$(C)$ for every $q_{1}$ and $q_{2}$ there exists a $q_{3}$ such that $q_{3} R q_{1}$ and $q_{3} R q_{2}$.

Our foundation thus consists of the system:

$\sum=\left(\mathfrak{A} ; \mathfrak{B} ; \mathfrak{M}^{\text {on } \mathfrak{P} \text { to } \mathfrak{x}, L} ; \quad N^{\text {on } \mathfrak{R} \text { to } \mathfrak{2} \geqq 0, \Delta} ; \mathfrak{M}^{\mathrm{Cp}} ; \mathfrak{Q} ; R^{\text {on } \mathfrak{D}, \pi \emptyset}\right)$.

3. The Fundamental Theorem. Our theorem concerns a set of operations $T_{q}$ on $\mathfrak{M}$ to $\mathfrak{A}$, which have the following properties:

$(\Delta=$ triangle $)$, for every $q, a_{1}, a_{2}, \mu_{1}, \mu_{2}$

$$
\left|T_{q}\left(a_{1} \mu_{1}+a_{2} \mu_{2}\right)\right| \leqq\left|a_{1} T_{q}\left(\mu_{1}\right)\right|+\left|a_{2} T_{q}\left(\mu_{2}\right)\right| ;
$$

$(M=$ modular or limited $)$, there exists an $M_{q}$ such that for every $\mu$

$$
\left|T_{q}(\mu)\right| \leqq M_{q} N \mu .
$$

We define as the modulus $M_{q}$ of $T_{q}$ the least upper bound of the values $\left|T_{q}(\mu)\right|$ for $N \mu=1$.

* The germ of such a general class $\mathfrak{M}$ is suggested by Riesz, Acta Mathematica, vol. 41 (1918), p. 72 . It has been used by Bennett, Proceedings of the National Academy, vol. 2 (1916), p. 595; Lamson, American Journal, vol. 42 (1920), p. 245; Hahn, Monatshefte, vol. 32 (1922), pp. 4 ff.; Banach, Fundamenta Mathematice, vol. 3 (1922), pp. $135 \mathrm{ff}$. 
The set $T_{q}$ is semi-uniformly bounded over $\mathfrak{M}$, ${ }^{*}$ i.e., there exists a sequence $\left\{q_{m}\right\}$ such that for every $\mu$ there exists an $m_{\mu}$ and an $e_{\mu}$ such that if $q R q_{m_{\mu}}$ then

$$
\left|T_{q}(\mu)\right| \leqq e_{\mu} \text {. }
$$

We note that on account of the properties $T$ and $C$ of $R$ the sequence $\left\{q_{m}\right\}$ may always be assumed to be monotonic increasing, i.e., for every $m q_{m} R q_{m-1}$. For if the property is holding for a given sequence $\left\{q_{m}\right\}$ it will also hold for the sequence $\left\{q_{m}{ }^{\prime}\right\}$, where $q_{1}{ }^{\prime}=q_{\mathrm{i}}$, and $q_{m}{ }^{\prime}$ is such that $q_{m}{ }^{\prime} R q_{m-1}^{\prime}$ and $q_{m}{ }^{\prime} R q_{m}$. Our theorem is as follows.

Fundamental Theorem. A set of operations $T_{q}$ on $\mathfrak{M}$ to $\mathfrak{A}$ having the properties $\Delta$ and $M$, which is semi-uniformly bounded over $\mathfrak{M}$, is ultimately uniformly limited, i.e., there exists a $q_{0}$ and an $M$ such that for $q R q_{0}$

$$
M_{q} \leqq M \text {. }
$$

This $q_{0}$ is a member of the sequence involved in the semi-uniform boundedness.

The proof of the theorem follows the lines of proofs of similar theorems in the literature. $\dagger$ Assume that the conclusion is not valid. Then the following statement would be true:

(A) For every $e>0$ and every $q_{m}$ of $\left\{q_{m}\right\}$ there exists a $q_{m e}$ such that $q_{m e} R q_{m}$ and $M_{q_{m e}}>e$.

Let $\left\{e_{n}\right\}$ be a sequence of numbers approaching infinity. Then by a step by step process we can determine $c_{n}, e^{(n)}, m_{n}$, $\mu_{n}, q_{e n}$, satisfying the following conditions:

(1) $c_{1}=1, c_{n}$ is the least of the quantities

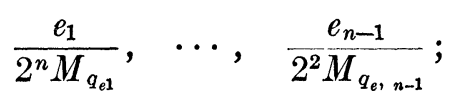

(2) $q_{e n}$ and $\mu_{n}$ such that $N \mu_{n}=1, q_{e n} R q_{m_{n-1}}$ and

$$
\left|T_{q_{e n}}\left(\mu_{n}\right)\right|>\frac{e_{n}+e^{(n-1)}}{c_{n}} ;
$$

(3) $m_{n}$ and $e^{(n)}$ such that $q R q_{m_{n}}$ implies $\left|T_{q}\left(\sum_{1}^{n} c_{i} \mu_{i}\right)\right|<e^{(n)}$.

${ }^{*}$ Cf. Moore and Smith, loc. cit., p. 114.

$\dagger$ Cf., for instance, Lebesgue, Annales de Toulouse, (3), vol. 1 (1909), p. 61; Hahn, loc. cit., p. 9; Banach, loc. cit., p. 157. 
The first of these is obviously possible. It insures the fact that $\Sigma_{1}^{\infty} c_{n}$ is convergent and

$$
\Sigma_{m}^{\infty} c_{n}<\frac{e_{m-1}}{2 M_{q_{\theta, m-1}}} .
$$

For (2) we apply statement $(A)$ to $q_{m_{n-1}}$ and $\left(e_{n}+e^{(n-1)}\right) / c_{n}$. The condition (3) follows from the semi-uniform boundedness of the set $T_{q}$. From (2) and (3) and from the property $\Delta$ of $T_{q}$ it follows that

$$
\begin{aligned}
\left|T_{q_{e n}}\left(\Sigma_{1}^{n} c_{i} \mu_{i}\right)\right| & \geqq|| T_{q_{e n}}\left(c_{n} \mu_{n}\right)|-| T_{q_{e n}}\left(\Sigma_{1}^{n-1} c_{i} \mu_{i}\right)|| \\
& >c_{n} \frac{e_{n}+e^{(n-1)}}{c_{n}}-e^{(n-1)} \\
& =e_{n} .
\end{aligned}
$$

Now since the series $\Sigma_{1}^{\infty} c_{n}$ is convergent, it follows that the sequence $\Sigma_{1}^{m} c_{n} \mu_{n}$ is a Cauchy sequence. For

$$
N\left(\Sigma_{k}^{m} c_{n} \mu_{n}\right) \leqq \Sigma_{k}^{m} c_{n} N \mu_{n}=\Sigma_{k}^{m} c_{n} \rightarrow 0 .
$$

Hence there exists a $\mu$ of the class $\mathfrak{M}$ such that

$$
\lim _{m} N\left(\mu-\Sigma_{1}^{m} c_{n} \mu_{n}\right)=0 .
$$

Then by the $\Delta$ property of $N$ we can show that

Hence

$$
N\left(\mu-\Sigma_{1}^{m} c_{n} \mu_{n}\right) \leqq \Sigma_{m+1}^{\infty} c_{n} .
$$

$$
\begin{aligned}
\left|T_{q_{e n}}(\mu)\right| & =\left|T_{q_{e n}}\left(\mu-\Sigma_{1}^{n} c_{i} \mu_{i}+\Sigma_{1}^{n} c_{i} \mu_{i}\right)\right| \\
& \geqq|| T_{q_{e n}}\left(\Sigma_{1}^{n} c_{i} \mu_{i}\right)|-| T_{q_{e n}}\left(\mu-\Sigma_{1}^{n} c_{i} \mu_{i}\right) \mid \\
& \geqq e_{n}-M_{q_{e n}} N\left(\mu-\Sigma_{1}^{n} c_{i} \mu_{i}\right) \geqq e_{n}-M_{q_{e n}} \Sigma_{n+1}^{\infty} c_{i} \\
& \geqq e_{n}-e_{n} / 2=e_{n} / 2,
\end{aligned}
$$

i.e., since $e_{n}$ approaches infinity with $n,\left|T_{q_{e n}}(\mu)\right|$ approaches infinity with $n$. But from the semi-uniform boundedness of $T_{q}$, it follows that there exists an $e_{\mu}$ and an $m_{\mu}$ such that for $q R q_{m_{\mu}}$ it is true that

$$
\left|T_{q}(\mu)\right|<e_{\mu} .
$$

Since the sequence $q_{m}$ is monotonic increasing and $q_{e n} R q_{m_{n-1}}$, it would follow that the sequence $\left|T_{q_{e n}}(\mu)\right|$ is bounded. We have thus reached a contradiction, showing that statement $(A)$ is untenable. Hence the theorem is proved. 
We can obtain various special cases of this theorem by replacing the hypotheses by special cases. If we assume, for instance, that $T_{q}(\mu)$ converges semi-uniformly over $\mathfrak{M}$,* then the set $T_{q}(\mu)$ is semi-uniformly bounded over $\mathfrak{M}$. Hence we have the following corollary.

Corollary I. If $T_{q}$ is a set of operations on $\mathfrak{M}$ to $\mathfrak{A}$ which have the properties $\Delta$ and $M$, for every $q$, and

$$
\lim _{q} T_{q}=T \text { (M); semi-uniformly), }
$$

then there exists a $q_{0}$ and an $M$ such that for $q R q_{0}$ it is true that

$$
M_{q} \leqq M
$$

where $M_{q}$ is the modulus of $T_{q}$. As a consequence $T$ also has the properties $\Delta$ and $M$ with modulus less than or equal to $M$.

If $T$ is linear, i.e., if for every $a_{1}$ and $a_{2}$, and $\mu_{1}$ and $\mu_{2}$

$$
T\left(a_{1} \mu_{1}+a_{2} \mu_{2}\right)=a_{1} T\left(\mu_{1}\right)+a_{2} T\left(\mu_{2}\right),
$$

then $T$ has the property $\Delta$, so that we have the following corollary.

CoRollary II. If $T_{q}$ is a set of linear limited operations on $\mathfrak{M}$ to $\mathfrak{A}$, which is semi-uniformly convergent over $\mathfrak{M}$ to $T$, then the set $T_{q}$ is ultimately uniformly limited and $T$ is a limited linear operation on $\mathfrak{M}$ to $\mathfrak{A}$.

Further specialization is possible by taking a particular class $\mathfrak{O}$. For instance suppose $\mathfrak{Q}$ is the class of positive integers. Then the set $T_{\cdot q}$ becomes a sequence of operations $T_{n}$ and the semi-uniform boundedness or convergence becomes simply boundedness or convergence, respectively, for every $\mu$. In this form the theorem includes a wide variety of cases, depending on the nature of the class $\mathfrak{M}$ and the norm $N$.

We obtain another corollary of this theorem by assuming that the $T_{q}$ are transformations on $\mathfrak{M}^{\prime}$ to $\mathfrak{M}^{\prime \prime}$, where $\mathfrak{M}^{\prime}$ and $\mathfrak{M}^{\prime \prime}$ are on $\mathfrak{P}^{\prime}$ and $\mathfrak{P}^{\prime \prime}$ respectively to $\mathfrak{A}$, and have the same properties as the class $\mathfrak{M}$, i.e., linearity, the existence of a norm with property $\Delta$ relative to which the classes are complete. It is possible to define the concepts limited or modular,

* Cf. Moore and Smith, loc. cit., p. 114.

$\dagger$ Hahn (loc. cit.) gives a large list of special classes and norms. The fact that a linear form, if convergent for every point of Hilbert space, is limited, is obviously also a special case of this theorem. 
and semi-uniform boundedness over $\mathfrak{M}^{\prime}$ for a set $T_{q}$ by replacing in the definition for operations on $\mathfrak{M}$ to $\mathfrak{A}$ the absolute value signs by the norm $N^{\prime \prime}$. Then we have the following corollary.

CoRollary III. If a set of linear limited transformations $T_{q}$ on $\mathfrak{M}^{\prime}$ to $\mathfrak{M}^{\prime \prime}$ is semi-uniformly bounded as to $\mathfrak{M}^{\prime}$, then the set is ultimately uniformly limited, i.e., there exists $a q_{0}$ and an $M$ such that $M_{q} \leqq M$ or $N^{\prime \prime} T_{q}\left(\mu^{\prime}\right) \leqq M N^{\prime} \mu^{\prime}$ for every $q R q_{0}$ and $\mu^{\prime *}$.

4. Bilinear Operations. An operation $B$ on $\mathfrak{M}^{\prime} \mathfrak{M}^{\prime \prime}$ to $\mathfrak{A}$, where $\mathfrak{M}^{\prime}$ and $\mathfrak{M}^{\prime \prime}$ have the properties of $\mathfrak{M}$, is bilinear if for every $\mu_{1}{ }^{\prime}, \mu_{2}{ }^{\prime}, \mu_{1}^{\prime \prime}, \mu_{2}^{\prime \prime}, a_{1}$ and $a_{2}$ it is true that

$$
\begin{aligned}
B\left(\mu_{1}^{\prime}+\mu_{2}^{\prime}, \mu_{1}^{\prime \prime}+\mu_{2}^{\prime \prime}\right)=B\left(\mu_{1}^{\prime}, \mu_{1}^{\prime \prime}\right)+B\left(\mu_{2}^{\prime}, \mu_{1}^{\prime \prime}\right) \\
\\
+B\left(\mu_{1}^{\prime}, \mu_{2}^{\prime \prime}\right)+B\left(\mu_{2}^{\prime}, \mu_{2}^{\prime \prime}\right),
\end{aligned}
$$

and

$$
B\left(a_{1} \mu_{1}^{\prime}, a_{2} \mu_{1}^{\prime \prime}\right)=a_{1} a_{2} B\left(\mu_{1}^{\prime}, \mu_{1}^{\prime \prime}\right) .
$$

It is limited or modular, if there exists a constant $M$ such that for every $\mu^{\prime}$ and $\mu^{\prime \prime}$

$$
\left|B\left(\mu^{\prime}, \mu^{\prime \prime}\right)\right| \leqq M N^{\prime} \mu^{\prime} N^{\prime \prime} \mu^{\prime \prime} .
$$

$M$ is called the modulus of $B$ when it is the least upper bound of $\left|B\left(\mu^{\prime}, \mu^{\prime \prime}\right)\right|$ for all $N^{\prime} \mu^{\prime}=1$ and $N^{\prime \prime} \mu^{\prime \prime}=1$. Then we have the following theorem.

THEOREM. A set of bilinear limited operations $B_{q}$ on $\mathfrak{M}^{\prime} \mathfrak{M}^{\prime \prime}$ to $\mathfrak{A}$ which is semi-uniformly bounded over $\mathfrak{M}^{\prime} \mathfrak{M}^{\prime \prime}$ is ultimately uniformly limited.

Let $T_{q}\left(\mu^{\prime}\right)$ be the least upper bound of $\left|B_{q}\left(\mu^{\prime}, \mu^{\prime \prime}\right)\right|$ for $N^{\prime \prime} \mu^{\prime \prime}=1$. This will exist for every $q$ and $\mu^{\prime}$ and will have the following properties:

$$
\begin{aligned}
T_{q}\left(a_{1} \mu_{1}^{\prime}+a_{2} \mu_{2}^{\prime}\right) & \leqq\left|a_{1} T_{q}\left(\mu_{1}^{\prime}\right)\right|+\left|a_{2} T_{q}\left(\mu_{2}^{\prime}\right)\right|, \\
T_{q}\left(\mu^{\prime}\right) & \leqq M_{q} N^{\prime} \mu^{\prime},
\end{aligned}
$$

* By specializing $\mathfrak{Q}, \mathfrak{M}^{\prime}$ and $\mathfrak{M}^{\prime \prime}$, we get a set of interesting theorems, including for instance some of interest in the definition of the summability for a series or integral. Cf., for instance, Kojima, TôHoku Journal, vol. 12 (1917), pp. $291 \mathrm{ff}$; Carmichael, this Bulletin, vol. 25 (1918), p. 118; Schur, Journal FÜr Mathematik, vol. 151 (1920), pp. $82 \mathrm{ff}$. 
the set $T_{q}\left(\mu^{\prime}\right)$ is semi-uniformly bounded. For if we consider $B_{q}\left(\mu^{\prime}, \mu^{\prime \prime}\right)$ as a function of $\mu^{\prime \prime}$ keeping $\mu^{\prime}$ fixed, we can apply the fundamental theorem. If $\left\{q_{m}\right\}$ is the sequence involved in the semi-uniform boundedness of $B_{q}$ then for every $\mu^{\prime}$, there will exist an $M_{\mu^{\prime}}$ and an $m_{\mu^{\prime}}$ such that for $q R q_{m_{\mu^{\prime}}}$ and every $\mu^{\prime \prime}$

$$
\left|B_{q}\left(\mu^{\prime}, \mu^{\prime \prime}\right)\right| \leqq M_{\mu^{\prime}} N^{\prime \prime} \mu^{\prime \prime} \quad \text { or } \quad T_{q}\left(\mu^{\prime}\right) \leqq M_{\mu^{\prime}} \text {. }
$$

It follows that $T_{q}\left(\mu^{\prime}\right)$ has the properties involved in the hypothesis of our fundamental theorem, and hence there exists an $m_{0}$ and an $M$ such that for $q R q_{m_{0}}$ and every $\mu^{\prime}$ it is true that

$$
T_{q}\left(\mu^{\prime}\right) \leqq M N^{\prime} \mu^{\prime} .
$$

Consequently for $q R q_{m_{0}}$ it is true that

$$
\left|B_{q}\left(\mu^{\prime}, \mu^{\prime \prime}\right)\right| \leqq T_{q}\left(\mu^{\prime}\right) N^{\prime \prime} \mu^{\prime \prime} \leqq M N^{\prime} \mu^{\prime} N^{\prime \prime} \mu^{\prime \prime}
$$

which is the ultimate uniform limitedness desired.

We can make specializations, as in the case of the fundamental theorem. The Hellinger-Toeplitz theorem is a special case of this theorem if $\mathfrak{M}^{\prime}=\mathfrak{M}^{\prime \prime}=$ Hilbert space, $\mathfrak{Q}$ is the class of all pairs of positive integers, and

$$
B_{q}\left(\mu^{\prime}, \mu^{\prime \prime}\right)=\Sigma_{1}^{n} \Sigma_{1}^{m} \beta_{i j} \mu_{i}^{\prime} \mu_{j}^{\prime \prime},
$$

where $\beta_{i j}$ are the coefficients of a bilinear form convergent for every $\mu^{\prime}, \mu^{\prime \prime}$ of Hilbert space. Obviously the sequence $q_{m}$ of the semi-uniform boundedness can be taken to be the set $(m, m)$ for all $m$.

Apparently, we can extend this theorem to that of a set of bilinear transformations on $\mathfrak{M}^{\prime} \mathfrak{M}^{\prime \prime}$ to $\mathfrak{M}^{\prime \prime \prime}$, or to a set of multilinear operations or transformations. From the latter it would be possible to derive the extension of the HellingerToeplitz theorem to multilinear forms.

The University of Michigan 